\title{
Retraction Note: Patients with ankylosing spondylitis treatment by golimumab: a systematic review and meta-analysis
}

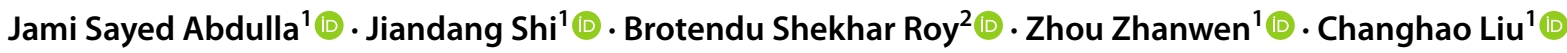

Published online: 14 February 2022

๑) Springer-Verlag GmbH Germany, part of Springer Nature 2022

Retraction Note: European Spine Journal (2020) 29:1813-1822 https://doi.org/10.1007/s00586-020-06466-9

The Editor-in-Chief has retracted this article because it overlaps with an unpublished manuscript by different authors that was submitted to another journal. The unpublished manuscript was submitted to the other journal before this article was submitted to the European Spine Journal.

ZhanWen Zhou agrees to this retraction. Jami Sayed Abdulla, Jiandang Shi, Brotendu Shekhar Roy and Changhao Liu have not responded to any correspondence from the publisher about this retraction.
Publisher's Note Springer Nature remains neutral with regard to jurisdictional claims in published maps and institutional affiliations.

The original article can be found online at https://doi.org/10.1007/ s00586-020-06466-9.

Jiandang Shi

shi_jiandang@outlook.com

Jami Sayed Abdulla

jami41@live.com

Brotendu Shekhar Roy

Pushpokroy@gmail.com

Zhou Zhanwen

270632413@qq.com

Changhao Liu

sylch1266@163.com

1 Department of Spinal Surgery, General Hospital of Ningxia Medical University, Ningxia Medical University, 804 Shengli Street, Xingqing District, Yinchuan 750004, Ningxia, People's Republic of China

2 First Affiliated Hospital of Nanchang University, No.17 Yong Wai Zheng Street Donghu District, Nanchang 330006, Jiangxi, People's Republic of China 